\title{
Prevalence of oral and oropharyngeal human papillomavirus in a sample of South African men: A pilot study
}

\author{
C L Davidson, ${ }^{1}$ BChD; K L Richter, ${ }^{2}$ MMed (Path) (Virology); M van der Linde, ${ }^{3} \mathrm{PhD}$; J Coetsee, ${ }^{3}$ MCom (Statistics); S C Boy, ${ }^{1} \mathrm{PhD}$ \\ ${ }^{1}$ Department of Oral Pathology and Oral Biology, School of Dentistry, Faculty of Health Sciences, University of Pretoria, South Africa \\ ${ }^{2}$ Department of Medical Virology, School of Medicine, Faculty of Health Sciences, University of Pretoria, and National Health Laboratory Service, \\ Tshwane Academic Division, Pretoria, South Africa \\ ${ }^{3}$ Department of Statistics, Faculty of Natural and Agricultural Sciences, University of Pretoria, South Africa
}

Corresponding author: C L Davidson (christy.davidson@up.ac.za)

\begin{abstract}
Background. Human papillomavirus (HPV) infection is well known to be associated with head and neck cancers (HNCs). HPV-associated HNCs are related to sexual behaviour, particularly the lifetime number of oral sex partners, but the epidemiology of oral and oropharyngeal HPV in South African men has not yet been studied.

Objectives. To determine the oral and oropharyngeal HPV strain prevalence and associated factors in a selected male population in Pretoria, South Africa (SA).

Methods. Male factory workers were recruited. Oral rinse and gargle samples were tested for $37 \mathrm{HPV}$ types using the Linear Array HPV Genotyping Test (Roche Molecular Systems). A questionnaire was used to obtain information regarding age, medical conditions, substance and alcohol use and sexual behaviour. HIV testing was optional.

Results. The HPV prevalence was 5.6\% among men (N=125) aged 17 - 64 years. High-risk HPV (hrHPV) types 16 and 68 were found in two men. Oral sex seemed to be an uncommon practice in the majority of respondents, but the two respondents with hrHPV did practise oral sex. There was a statistically significant association between HPV infection and an increased number of sexual partners ( $p=0.027)$, but not between HPV and substance use, HIV status or clinical mucosal pathology.

Conclusion. The prevalence of oral and oropharyngeal HPV was lower than reported in other countries. An association between oral HPV and having multiple sexual partners was found. A larger nationwide study would give a more representative view of the burden of oral and oropharyngeal HPV infection in SA.
\end{abstract}

S Afr Med J 2014;104(5):358-361. DOI:10.7196/SAMJ.7542

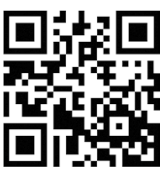

Oropharyngeal squamous cell carcinoma (OSCC) may originate in the soft palate, tongue base, pharyngeal walls or tonsils. The association of high-risk human papillomavirus (hrHPV) types with increased risk for a subset of OSCC has been established by many studies. ${ }^{[1-5]}$ It has been shown that oral human papillomavirus (HPV) infection, and specifically HPV type 16 infection, causes an up to 50 -fold increase in HPV-positive OSCC. ${ }^{[6]}$ The pathogenic association of OSCC with HPV was therefore recently accepted by the International Agency for Research on Cancer. ${ }^{[7]}$ Head and neck cancer (HNC), of which OSCC is a subset, is the sixth most common cancer in the world. In South Africa (SA) there has been a proven increase in OSCC since 1995 , and $90 \%$ of these cancers were associated with HPV $16 .{ }^{[8]}$ Males were affected more frequently than females. ${ }^{[8]}$ Gillison et al. ${ }^{[9]}$ recently published the first population-based study that concurrently examined the epidemiology of oral HPV infection among males and females and showed an almost three-fold higher HPV prevalence in males; the HPV 16 prevalence was five-fold higher in males than in females.

The aims of this pilot study were to determine the oral and oropharyngeal HPV strain prevalence in a selected male population in Pretoria, SA, as well as the association of HPV with sexual practices and substance use.

\section{Methods \\ Participant selection}

Male participants were voluntarily recruited from an industrial factory in Pretoria in 2012. Written informed consent was obtained from each participant for HPV testing and answering the questionnaire. Separate consent was obtained for voluntary HIV testing. Refusal of HIV testing did not serve as a criterion for exclusion from the study, but refusal of HPV testing did. Approval for the study was obtained from the Ethics Committee of the Faculty of Health Sciences, University of Pretoria (no. 101/2012).

\section{Clinical examination and saliva sample collection}

Participants underwent a thorough oral and oropharyngeal clinical examination by a senior dentist/oral pathologist. All dental and other soft-tissue abnormalities were noted and photographed.

Oral rinse samples were collected by means of a 20 -second oral rinse and gargle with $5 \mathrm{ml}$ phosphate-buffered saline (PBS) (Sigma Life Sciences, USA) given to the patient in a $40 \mathrm{ml}$ sterile collection cup (Lasec, SA). Participants alternately swished and gargled and then expectorated the saliva into the cup. All samples were numbered consecutively and stored on ice until sample preparation. 


\section{Questionnaire}

The questionnaire comprised specifically formulated questions pertaining to past and present sexual histories, substance and alcohol use and any medical conditions. The numbers of the questionnaires corresponded with the oral rinse samples, and their content was treated as confidential.

\section{Voluntary HIV counselling and testing}

Participants who opted for HIV testing received pre-test counselling from the occupational health practitioner (OHP) of the company. A rapid point-of-care HIV screening test (U test HIV/AIDS, Humor Diagnostica, SA) that detects HIV-1 and HIV-2 antibodies was then performed according to the manufacturer's instructions. Confirmatory follow-up testing and continued care were conducted by the same OHP.

\section{Molecular laboratory techniques Specimen preparation}

Saliva samples were prepared for HPV genotyping within 24 hours of collection. The samples were washed three times with sterile PBS and the cell pellets were stored at $-70^{\circ} \mathrm{C}$ until subsequent DNA extraction.

\section{DNA extraction}

DNA extraction from the cell pellets was performed using the DNA Isolation Kit (Roche Molecular Systems, USA) on the MagNA Pure automated extraction system.

\section{HPV DNA amplification and genotyping}

HPV DNA amplification and genotyping was done using the Linear Array HPV Genotyping Test (Roche Molecular Systems). The pool of primers in the kit are designed to amplify HPV DNA from 15 high-risk genotypes (16, $18,31,33,35,39,45,51,52,56,58,59,68,73$ and 82 ), 3 probable high-risk genotypes (26, 53 and 66) and 19 low/undetermined risk types $(6,11,40,42,54,55,61,62,64,67,69$, 70, 71, 72, 81, 83, 84, IS39 and CP6108)..$^{[10]}$ The $\beta$-globin gene was amplified concurrently for each sample and served as internal control for cellular adequacy, extraction efficiency and amplification.

\section{Statistical analysis}

The relationship between the prevalence of HPV and the prevalence of the variables substance use, alcohol use, sexual history, HIV and oral lesions was investigated using the chi-square test for independence or, where appropriate, Fisher's exact test. The Kruskal-Wallis test was used to analyse whether or not any of the variables

Table 1. Summary of human papillomavirus status and results for the variables investigated in all the participants

\begin{tabular}{|c|c|c|c|c|}
\hline & $\begin{array}{l}\mathrm{HPV}+(N=7) \\
n(\%)\end{array}$ & $\begin{array}{l}\text { HPV- }(N=118) \\
n(\%)\end{array}$ & $\begin{array}{l}\text { Total }(N=125) \\
n(\%)\end{array}$ & $p$-value \\
\hline Age (years) & & & & 1.000 \\
\hline$\leq 40$ & $2(28.6)$ & $36(30.5)$ & $38(30.4)$ & \\
\hline$>40$ & $5(71.4)$ & $82(69.5)$ & $87(69.6)$ & \\
\hline Smoking & & & & 0.703 \\
\hline Yes & $4(57.1)$ & $54(45.8)$ & $58(46.4)$ & \\
\hline No & $3(42.9)$ & $64(54.2)$ & $67(53.6)$ & \\
\hline Alcohol & & & & 0.343 \\
\hline Yes & $7(100.0)$ & $93(78.8)$ & $100(80.0)$ & \\
\hline No & 0 & $25(21.2)$ & $25(20.0)$ & \\
\hline Lifetime sexual partners & & & & 0.027 \\
\hline $0-5$ & $1(14.3)$ & $19 / 117^{*}(16.2)$ & $20 / 124(16.1)$ & \\
\hline $5-20$ & $2(28.6)$ & $79 / 117^{*}(67.5)$ & $81 / 124(65.3)$ & \\
\hline$>20$ & $4(57.1)$ & $19 / 117^{*}(16.2)$ & $23 / 124(18.5)$ & \\
\hline $\begin{array}{l}\text { Sexual partners in the past } \\
6 \text { months }\end{array}$ & & & & 1.000 \\
\hline None & $0(0)$ & $3 / 117(2.6)$ & $3 / 124(2.4)$ & \\
\hline $1-3$ & $7(100)$ & $104 / 117(88.9)$ & $111 / 124(89.5)$ & \\
\hline$>3$ & $0(0)$ & $10 / 117(8.5)$ & $10 / 124(8.1)$ & \\
\hline Practise oral sex & & & & 0.525 \\
\hline Yes & $3(42.9)$ & $48(40.7)$ & $51(40.8)$ & \\
\hline No & $4(57.1)$ & $70(59.3)$ & $74(59.2)$ & \\
\hline Lifetime oral sex partners & & & & 0.592 \\
\hline 1 & 0 & $19 / 48(39.6)$ & $19 / 51(37.3)$ & \\
\hline 2 & $1 / 3(33.3)$ & $16 / 48(33.3)$ & $17 / 51(33.3)$ & \\
\hline$>2$ & $2 / 3(66.7)$ & $17 / 48(35.4)$ & $19 / 51(37.3)$ & \\
\hline $\begin{array}{l}\text { Oral sex partners in the past } \\
6 \text { months }\end{array}$ & & & & 0.518 \\
\hline None & $4(57.1)$ & $83(70.3)$ & $87(69.6)$ & \\
\hline 1 & $2(28.6)$ & $26(22.0)$ & $28(22.4)$ & \\
\hline$>1$ & $1(14.3)$ & $9(7.6)$ & $10(8.0)$ & \\
\hline HIV & & & & 0.563 \\
\hline Positive & 0 & $5(4.2)$ & $5(4.0)$ & \\
\hline Negative & $4(57.1)$ & $81(68.6)$ & $85(68.0)$ & \\
\hline Declined testing & $3(42.9)$ & $32(27.1)$ & $35(28.0)$ & \\
\hline Clinical lesions & & & & 0.592 \\
\hline With & 0 & $18(15.3)$ & $18(14.4)$ & \\
\hline Without & $7(100.0)$ & $100(84.7)$ & $107(85.6)$ & \\
\hline $\begin{array}{l}\mathrm{HPV}+=\text { HPV-positive; HPV }=\text { HPV-1 } \\
{ }^{*} \text { One questionnaire was not completed } \\
\\
\text { "Fisher's exact test. }\end{array}$ & & & & \\
\hline
\end{tabular}

investigated had any significant influence on the HPV status of the participants.

\section{Results}

Oral rinse specimens were collected from 128 males between the ages of 17 and 64 years (median 50). The participants originated from all over SA. Three participants who did not complete questionnaires were excluded from the study. Only $7 / 125$ participants (5.6\%) tested positive for HPV. One participant was co-infected with HPV types 71 and 72 . Two 
Table 2. Summary of human papillomavirus-positive results for all the variables investigated

\begin{tabular}{lllllllll}
\hline & HPV type/s & Age (years) & Smoking & Alcohol & Sex & Oral sex & HIV status & Oral lesions \\
\hline 1 & $16(\mathrm{hr})$ & 51 & Yes & Yes & Yes $(\mathrm{m})$ & Yes & Declined testing & No \\
2 & 55 & 59 & Yes & Yes & Yes (m) & No & Declined testing & No \\
3 & 62 & 46 & No & Yes & Yes (m) & No & Negative & No \\
4 & $68(\mathrm{hr})$ & 58 & No & Yes & Yes & Yes & Negative & No \\
5 & 70 & 29 & Yes & Yes & Yes (m) & No & Declined testing & No \\
6 & 71,72 & 64 & No & Yes & Yes (m) & No & Negative & No \\
7 & 72 & Yes & Yes & Yes (m) & Yes & Negative & No \\
HPV = human papillomavirus; hr = high-risk HPV type; $\mathrm{m}=$ multiple sexual partners. & & & & & &
\end{tabular}

participants tested positive for hrHPV types, namely HPV 16 and HPV 68 , respectively. Table 1 summarises the findings for all the variables investigated. The quantity and frequency of the different substances used were not investigated. An overall score was calculated, which only reflected whether a respondent used or did not use any of the substances listed in the questionnaire.

All participants were sexually active, 90/125 (72.0\%) of them reporting having had their first sexual encounter between 15 and 20 years of age. The association between HPV and the number of sexual partners was statistically significant $(p=0.027)$.

In the subgroup of 90 men $(72.0 \%)$ who volunteered for HIV testing, only five (5.5\%) tested positive. Unfortunately only 4/7 HPVpositive participants (57.1\%) opted for HIV testing; one of these was found to have an hrHPV type. All the HPV-positive participants who tested for HIV were negative.

Only 18 of the 125 participants (14.4\%) were found to have oral mucosal lesions on clinical examination. The lesions encountered included white lesions, red lesions, ulcerative lesions and Candida albicans infection. None of the HPV-positive participants had clinically visible mucosal pathology.

Table 2 summarises the findings for the variables investigated in the HPV-positive participants.

\section{Discussion}

The natural history of oral and oropharyngeal HPV infection in men is unknown. A recent study found the prevalence of oral HPV in men in the USA to be around $10 \% .{ }^{[9]}$ This pilot study among a group of SA men had an unexpectedly low HPV prevalence of only $5.6 \%$. This could be attributed to the small sample size, or to the observation that oral sex did not seem to be a common practice among the group of men studied. A much larger nation-wide analysis with interinstitutional collaboration will be necessary to confirm whether this figure is in fact truly representative of oral and oropharyngeal HPV in SA men. Known risk factors for oral and oropharyngeal HPV infection currently include lifetime number of sexual partners, ${ }^{[6,9,11]}$ number of oral sex partners, ${ }^{[1,12]}$ substance use ${ }^{[9,12-14]}$ and infection with HIV. ${ }^{[15-18]}$

Sexual contact, either genital or oral, remains the primary mode of HPV transmission, and it is well documented that having multiple partners increases the prevalence of oral and genital HPV.$^{[6,9,19,20]} \mathrm{A}$ study in KwaZulu-Natal, SA, reported male undergraduate students to have had a median of four sexual partners in the previous year. ${ }^{[1]}$ In many African cultures it is acceptable for men to have multiple partners and engage in sex outside the primary relationship. ${ }^{[21,22]} \mathrm{A}$ statistically significant association between the number of sexual partners and the presence of oral HPV was seen in this study ( $p=0.027$ ), with $4 / 7$ HPV-positive participants having had $>20$ sexual partners and 2/7 having had between five and 20 partners. The numbers of sexual partners of the HPV-positive participants in the past 6 months ranged from one to three.

Tobacco use is an accepted cause of HNCs, but it is not considered a strong risk factor for HPV-associated oropharyngeal cancers. ${ }^{[1,6]} \mathrm{We}$ found no statistically significant association between smoking and the presence of HPV ( $p=0.703$ ), although the only participant with HPV 16 in our study was a current cigarette smoker. An increased oral HPV prevalence has been shown to be associated with current and previous smoking, but the exact pathogenic mechanisms are unknown. ${ }^{[12,13]}$

Alcohol has the ability to modify mucosal tissue, allowing easier entry of the virus, and is also known to modify the host immune response, thereby increasing susceptibility to HPV infection. ${ }^{[14,23]}$ In our study we found no statistical association between alcohol use and HPV ( $p=0.343)$, although all the HPV-positive participants were current alcohol users.

The HIV pandemic and attempts to prevent spread of the virus have increasingly highlighted sexual practices other than penetrative sex. Oral sex has increased to a marked extent, especially among teenagers, as a result of the false perception that sexually transmitted diseases such as HIV infection cannot be passed on in this manner. ${ }^{[12,24]}$ Studies on oral sex practices in the USA have shown that 19.6 - 78\% of young adults had engaged in oral sex in their lifetime. ${ }^{[25]}$ Gillison et al. ${ }^{[9]}$ in line with other studies, found oral sex performed on women to be one possible explanation for the increased prevalence of oropharyngeal HPV in males, who in their study on average had more partners than the females. Sexual behaviour studies in SA are few, and those that do exist are not specific in their data regarding genital versus oral sexual practices and the gender differences that exist. However, it was later discovered through the male fieldworker that most men in our study interpreted oral sex to mean contact of the female's oral mucosa with the man's genital mucosa rather than the other way around. This could explain the low prevalence of oral HPV. Only 51 of all participants (40.8\%) and 3/7 HPV-positive participants $(42.9 \%)$ answered 'yes' to the question whether they had practised oral sex. Considering the participants' understanding of the definition of oral sex, one should be cautious in interpreting these figures. Of note was that both the individuals with hrHPV types said that they had had oral sex with more than one partner.

Oral HPV infection is considered to be bimodal in age distribution, with a high prevalence in younger men (30 - 34 years of age) and in later years $(60-64){ }^{[9]}$ The participants who were HPV-positive in this study were between 29 and 64 years of age, and those with hrHPV were between 50 and 60 years of age. It has been speculated that the higher HPV prevalence at older ages could be due to increased duration of infections at older ages, rather than an increased 
acquisition of new HPV infections. ${ }^{[26]}$ In our study, however, all the participants who were positive for HPV had had between one and three sexual partners in the past 6 months, suggesting that their HPV infections could have been newly acquired.

HIV-infected individuals have been reported to have an up to six-fold increased risk for HPV-related OSCC. ${ }^{[27]}$ It is thought that HIV and HPV function as a combined risk factor, as HIV-positive individuals have more frequent HPV infections, but the natural history of these infections may be altered when they co-exist. ${ }^{[15]}$ In SA, the total estimated number of adults aged 15 - 49 years living with HIV in 2013 was 5.26 million, giving an HIV prevalence of $10 \% .{ }^{[28]}$ The HIV prevalence in this study was only $5.5 \%$. Only four of the HPV-positive participants $(n=7)$ volunteered to test for HIV, and all were negative. One of the participants who was found to have hrHPV 16, which is associated with OSCC, declined HIV testing. A much larger nationwide study with an HIV prevalence more representative of that for the country as a whole will be needed to determine the role of HIV infection in the natural history of oral and oropharyngeal HPV infection.

The 18 participants who presented with oral mucosal lesions were all negative for HPV. All patients with dental or mucosal pathology were referred to peripheral dental clinics or the Oral Medicine Clinic of the Oral and Dental Hospital, Pretoria, for surgical biopsies and clinical follow-up.

\section{Study strength}

Ours is the first study of this nature to investigate the oral and oropharyngeal HPV prevalence in SA men. Although the sample size was small, we did find a statistical association between HPV and the number of lifetime sexual partners.

\section{Study limitations}

The greatest limitation of this study is the small sample size, which was restricted owing to funding. Another limitation is the fact that not all the participants opted to test for HIV, which may also have an influence on the conclusions. Because this was a pilot study some of the conclusions made, including the role of HIV, will need to be verified by a larger, nationwide study.

\section{Conclusion}

In contrast to the prevalence of genital HPV in SA females and the role of the virus in the pathogenesis of cervical carcinoma, little is known about the prevalence of HPV in the oropharyngeal mucosa of SA men, who are known to acquire HPV-related OSCC more frequently than women. The prevalence of oral and oropharyngeal HPV in this study is lower than reported in other countries. Even though the number of participants was low, a statistically significant association was shown between oral and oropharyngeal HPV and having multiple sexual partners. Oral sex, although not practised by the majority of participants, was practised by those identified with hrHPV types. The high number of deaths from HPV-related cervical carcinoma and the increase in HPV-related OSCC, especially in males, makes it urgently necessary to investigate the prevalence of oral and oropharyngeal HPV in the larger SA population. Specific reference to the roles of sexual practices and HIV in the natural history of HPV is essential. Deeper investigations into the nature of sexual practices among South Africans are needed, and the diversity of the SA population will have to be considered in any future study of this nature.
Acknowledgements. We thank Dental Warehouse for the generous donation of the materials used for the oral rinse and gargle collection, the Dental Research Education and Development Trust of the South African Dental Association for funding, Professor A Ligthelm, Dean of the School of Dentistry, Faculty of Health Sciences, University of Pretoria, and Professor M Pepper, Director of the Institute for Cellular and Molecular Medicine at the University. We also thank Matron Ella Khumalo and Mr S Mafiri for their valuable assistance with the sampling and Sister Marina Jordaan for all her assistance with and organisation of the study.

\section{References}

1. D'Souza G, Kreimer AR, Viscidi R, et al. Case-control study of human papillomavirus and oropharyngeal cancer. N Engl J Med 2007;356(19):1944-1956. [http://dx.doi.org/10.1056/NEJMoa065497]

cancer. N Engl] Med 2007;356(19):1944-1956. [http://dx.doi.org/10.1056/NEJMoa065497]
2. Gillison M. Human papillomavirus-related diseases: Oropharynx cancers and potential implications for 2. Gillison M. Human papillomavirus-related diseases: Oropharynx cancers and potential implications for
adolescent HPV vaccination. J Adolesc Health 2008;43(4 Suppl):S52-S60. [http://dx.doi.org/10.1016/j. jadohealth.2008.07.002

3. Andrews E, Seaman WT, Webster-Cyriaque J. Oropharyngeal carcinoma in non-smokers and non-drinkers: A role for HPV. Oral Oncol 2009;45(6):486-491. [http://dx.doi.org/10.1016/j.oraloncology.2008.07.008]

4. Kreimer AR, Clifford GM, Boyle P, Franceschi S. Human papillomavirus types in head and neck squamous cell carcinomas worldwide: A systematic review. Cancer Epidemiol Biomarkers Prev 2005;14(2):467-475. [http:// dx.doi.org/10.1158/1055-9965.EPI-04-0551]

5. Pannone G, Santoro A, Papagerakis S, Lo Muzio L, De Rosa G, Bufo P. The role of human papillomavirus in the pathogenesis of head \& neck squamous cell carcinoma: An overview. Infect Agent Cancer 2011;6:4. [http:// dx.doi.org/10.1186/1750-9378-6-4]

6. Gillison ML, D'Souza G, Westra W, et al. Distinct risk factor profiles for human papillomavirus type 16-positive and human papillomavirus type 16-negative head and neck cancers. J Natl Cancer Inst 2008;100(6):407-420.
and and human papillomavirus type 16-neg

7. International Agency for Research on Cancer. Human papillomaviruses: IARC monographs working group 7. International Agency for Research on Cancer. Human papillomaviruses: IARC monographs working group
on the evaluation of carcinogenic risks to humans. 2007. http://www.monographs.iarc.fr/ENG/Monographs/ vol90 (accessed 22 August 2013)

8. WHO/ICO Information Centre on HPV and Cervical Cancer. Human papillomavirus and related cancers in South Africa. Summary report 2010. http://www.who.int/hpvcentre (accessed 22 August 2013).

9. Gillison ML, Broutian T, Pickard RKL, et al. Prevalence of oral HPV infection in the United States, 2009-2010. JAMA 2012;307(7):693-703. [http://dx.doi.org/10.1001/jama.2012.101]

10. Munoz N, Bosch FX, de Sanjose S, et al. Epidemiologic classification of human papillomavirus types associated with cervical cancer. N Engl J Med 2003;348(6):518-527. [http://dx.doi.org/10.1056/NEJMoa021641]

11. Hoque ME. Sexual practices among male undergraduate students in KwaZulu-Natal, South Africa. Southern African Journal of Epidemiology and Infection 2011;26(3):157-160. [http://dx.doi.org/10.4102/phcfm. v3i1.281]

12. D’Souza G, Agrawal Y, Halpern J, Bodison S, Gillison ML. Oral sexual behaviours associated with prevalent oral human papillomavirus infection. J Infect Dis 2009;199(9):1263-1269. [http://dx.doi.org/10.1086/597755]

13. Kreimer AR, Villa A, Nyitray AG, et al. The epidemiology of oral HPV infection among a multinational sample Kreimer AR, Villa A, Nyitray AG, et al. The epidemiology of oral HPV infection among a multinational sample
of healthy men. Cancer Epidemiol Biomarkers Prev 2011;20(1):172-182. [http://dx.doi.org/10.1158/1055of healthy men. Ca

14. Smith EM, Ritchie JM, Summersgill KF, et al. Human papillomavirus in oral exfoliated cells and risk of head and neck cancer. J Natl Cancer Inst 2004; 96(6):449-455. [http://dx.doi.org/10.1093/jnci/djh233]

15. Steinau M, Reddy D, Sumbry A, et al. Oral sampling and human papillomavirus genotyping in HIV-infected patients. J Oral Pathol Med 2012;41 (4):288-291. [http://dx.doi.org/10.1111/j.1600-0714.2011.01093.x]

16. Beachler DC, Weber KM, Margolick JB, et al. Risk factors for oral HPV infection among a high prevalence population of HIV-positive and at-risk HIV-negative adults. Cancer Epidemiol Biomarkers Prev 2012;21(1):122-133. [http://dx.doi.org/10.1158/1055-9965.EPI-11-0734]

17. Marais D, Passmore J-AS, Denny L, Sampson C, Allan B, Williamson A-L. Cervical and oral human papillomavirus types in HIV-1 positive and negative women with cervical disease in South Africa. J Med Virol 2008;80(6):953-959. [http://dx.doi.org/10.1002/jmv.21166]

18. Fakhry C, D’Souza G, Sugar E, et al. Relationship between prevalent oral and cervical human papillomavirus infections in human immunodeficiency virus-positive and -negative women. J Clin Microbiol 2006;44(12):4479-4485. [http://dx.doi.org/10.1128/JCM.01321-06]

19. Schwartz S, Daling J, Doody D, et al. Oral cancer risk in relation to sexual history and evidence of 9. Schwartz S, Daling J, Doody D, et al. Oral cancer risk in relation to sexual history and evidence of
human papillomavirus infection. J Natl Cancer Inst 1998;90(21):1626-1636. [http://dx.doi.org/10.1093/ human papillo

jnci/90.21.1626] . Hernandez BY, Wilkens LR, Zhu X, et al. Transmission of human papillomaviru
Emerg Infect Dis 2008;14(6):888-894. [http://dx.doi.org/10.3210/eid1406.0706162]

21. Ackermann L, Klerk G. Social factors that make South African women vulnerable to HIV infection. Health Care Women Int 2002;23(2):163-172. [http://dx.doi.org/10.1080/073993302753429031]

22. Jewkes R, Morrell R. Gender and sexuality: Emerging perspectives from the heterosexual epidemic in South Africa and implications for HIV risk and prevention. J Int AIDS Soc 2010;13(6):1-11. [http://dx.doi. org/10.1186/1758-2652-13-6]

23. Molina P, McClain C, Valla D, Guidot D, Diehl A, Lang C. Molecular pathology and clinical aspects of alcoholinduced tissue injury. Alcohol Clin Exp Res 2002;26(1):120-128. [http://dx.doi.org/10.1111/j.1530-0277.2002. tb02440.x]

24. Cherie A, Berhane Y. Oral and anal sex practices among high school youth in Addis Ababa, Ethiopia. BMC Public Health 2012;12:5. [http://dx.doi.org/10.1186/1471-2458-12-5]

25. Ompad DC, Strathdee SA, Celentano DD, et al. Predictors of early initiation of vaginal and oral sex among urban young adults in Baltimore, Maryland. Arch Sex Behav 2006;35(1):53-65. [http://dx.doi.org/10.1007/
uration s10508-006-8994-x]

26. Kreimer AR, Pierce Campbell CM, Lin HY, et al. Incidence and clearance of oral human papillomavirus infection in men: The HIM cohort study. Lancet 2013;382(9895):877-887. [http://dx.doi.org/10.1016/S01406736(13)60809-0

27. Engels EA, Biggar RJ, Hall HI, et al. Cancer risk in people infected with human immunodeficiency virus in the United States. Int J Cancer 2008;123(1):187-194. [http://dx.doi.org/10.1002/ijc.23487]

28. Statistics South Africa. Mid-year population estimates 2013. http://www.statssa.gov.za/publications/P0302/ P03022013.pdf (accessed 22 August 2013).

Accepted 22 November 2013. 